\title{
Vergleich von vier baugleichen elektronischen Nasen und drei Messaufbauten
}

\section{Comparison of Four Identical Electronic Noses and Three Measurement Set-Ups}

Autoren

Institute
R. Koczulla' , A. Hattesohl' ${ }^{1}$, H. Biller ${ }^{2}$, J. Hofbauer ${ }^{3}$, J. Hohlfeld ${ }^{2}$, C. Oeser ${ }^{4}$, H. Wirtz ${ }^{4^{*}}$, R. A. Jörres ${ }^{3^{*}}$

Klinik für Innere Medizin, Schwerpunkt Pneumologie, Philipps-Universität Marburg

Abteilung Klinische Atemwegsforschung, Fraunhofer Institut für Toxikologie und Experimentelle Medizin, Hannover

3 Institut und Poliklinik für Arbeits-, Sozial- und Umweltmedizin, Ludwig-Maximilians-Universität München

${ }_{4}^{4}$ Abteilung Pneumologie, Department Innere Medizin, Universität Leipzig eingereicht 23.12.2010 akzeptiert nach Revision 7.2. 2011

\section{Bibliografie}

Dol http://dx.doi.org/ 10.1055/s-0030-1256280

Online-Publikation: 24. 3. 2011

Pneumologie 2011; 65:

465-470 @ Georg Thieme

Verlag KG Stuttgart · New York ISSN 0934-8387

\section{Korrespondenzadresse}

Dr. A. R. Koczulla

Universitätsklinikum Gießen und Marburg

Abteilung für Pulmonologie

Standort Marburg

Baldingerstraße 1

35042 Marburg

koczulla@med.uni-marburg.de

\section{Zusammenfassung \\ $\nabla$}

Hintergrund: Volatile organische Komponenten (VOCs) agieren als Biomarker in der Ausatemluft und können mithilfe sog. elektronischer Nasen (eNose) detektiert werden. Die VOC-Profile können bioinformatisch miteinander verglichen werden. Jedoch ist nicht eruiert, inwieweit Sensoren von Geräten gleichen Typs identisch messen und unterschiedliche Messaufbauten sowie die Feuchtigkeit der Luft das VOC-Profil beeinflussen. Angesichts des großen Interesses an eNose-Verfahren sind dies wichtige Vorinformationen für eine eventuelle Anwendung.

Methodik: Mit drei typischen, unterschiedlichen Messaufbauten wurden jeweils drei Probanden mit insgesamt vier Geräten gleichen Typs (Cyranose 320, Smiths Detection) gemessen. Die Ausatemluft wurde ungefiltert in einem Sammelbeutel aufgenommen. Als Referenzluft wurde zum einen unbehandelte Raumluft und in zwei Aufbauten zu 100\% befeuchtete Luft verwendet. In einem der letztgenannten Aufbauten atmeten die Probanden vor der Messung Raumluft, in dem anderen hochreine Luft. Die Daten wurden sowohl mittels der Originalzeitsignale als auch der vom Gerät gelieferten Widerstandsänderungen ausgewertet.

Ergebnisse: Es zeigten sich unterschiedliche Ergebnisse, je nach Aufbau. Das Anfeuchten der Referenzluft konnte die Varianz zwischen den Geräten reduzieren, doch hing das Ergebnis vom Auswerteverfahren ab. Beim Vergleich der Probanden ergab sich, dass Aufbauten mit Befeuchtung die Probanden ähnlich abbildeten, hingegen bei Verwendung von Raumluft als Referenz die Signale, aber auch die Streuung größer waren.

\footnotetext{
* Die Autoren H. Wirtz und R. A. Jörres waren gleichmäßig beteiligt.
}

\section{Abstract \\ $\nabla$}

Background: Volatile organic compounds (VOCs) can be used as biomarkers in exhaled air. VOC profiles can be detected by an array of nanosensors of an electronic nose. These profiles can be analysed using bioinformatics. It is, however, not known whether different devices of the same model measure identically and to which extent different set-ups and the humidity of the inhaled air influence the VOC profile.

Methods: Three different measuring set-ups were designed and three healthy control subjects were measured with each of them, using four devices of the same model (Cyranose $320^{\mathrm{TM}}$, Smiths Detection). The exhaled air was collected in a plastic bag. Either ambient air was used as reference (set-up Leipzig), or the reference air was humidified (100\% relative humidity) (set-up Marburg and set-up Munich). In the set-up Marburg the subjects inhaled standardised medical air (Aer medicinalis Linde, AGA AB) out of a compressed air bottle through a demand valve; this air (after humidification) was also used as reference. In the set-up Leipzig the subjects inhaled VOC-filtered ambient air, in the set-up Munich unfiltered room air. The data were evaluated using either the real-time data or the changes in resistance as calculated by the device.

Results: The results were clearly dependent on the set-up. Apparently, humidification of the reference air could reduce the variance between devices, but this result was also dependent on the evaluation method used. When comparing the three subjects, the set-ups Munich and Marburg mapped these in a similar way, whereas not only the signals but also the variance of the set-up Leipzig were larger. 
Schlussfolgerungen: Die Messung mittels eNose ist bislang nicht vereinheitlicht, und der Aufbau beeinflusst die Ergebnisse erheblich. Da andere Untersucher noch weitere Verfahren einsetzen, sind zurzeit keine allgemeingültigen Schlüsse möglich. Es bedarf systematischer Tests, um den sensitivsten, zuverlässigsten und zugleich noch praktikablen Aufbau zu finden und so die Vergleichbarkeit zu erreichen.

\section{Hintergrund \\ $\nabla$}

Die Diagnosestellung mithilfe von Gerüchen hat eine lange Tradition in der Medizin. Der Foetor hepaticus, der putride Geruch anaerober Infektionen und das ketoazidotische Koma sind klassische Beispiele, bei denen ein typischer Geruch zur Diagnose führt. Technologische Fortschritte bei chemischen Sensoren, in der Datenverarbeitung, der Generierung lernfähiger Systeme und im Erstellen diagnostischer Algorithmen machen den nichtinvasiven Ansatz der olfaktorischen Diagnostik zusehends interessanter. So wurden in den 90er-Jahren sogenannte „elektronische Nasen“ (eNose) entwickelt. Diesen liegen verschiedene technische Prinzipien zugrunde.

Unter anderem werden Zusammenstellungen (Arrays) multipler Sensoren verwendet, deren Signale im Sinne einer Mustererkennung verarbeitet werden. Diese Sensoren funktionieren nicht wie Riechzellen, dennoch sind Ähnlichkeiten vorhanden. Riechzellen enthalten Rezeptormoleküle, die mit wenigen Gasen bzw. funktionellen Gruppen wechselwirken und so ein Signal erzeugen. Sie sind allerdings nicht selektiv für nur eine einzige der möglichen Substanzen; daher wird im Gehirn ein multivariates Erregungsmuster verschiedenartiger Riechzellen zur Erkennung verwendet. Zugleich findet innerhalb der Zellen sowie durch Zusammenfassung gleichartiger Zellen eine Signalverstärkung statt, sodass auch niedrige Gaskonzentrationen zur Detektion ausreichen.

Bei eNose-Geräten binden volatile organische Komponenten (VOCs) aufgrund von Größe, Dipolcharakter und Wasserstoffbindekapazität reversibel an die Sensoren. Diese sind sensitiv gegenüber physikochemisch charakterisierten Substanzklassen, sodass überlappende Bindungen die Regel sind. Dennoch differieren sie aufgrund ihrer unterschiedlichen Dotierung im Muster der relativen Aktivierung. Die Unterscheidung zwischen Substanzen oder Substanzgemischen erfolgt mittels Verfahren der multivariaten Musteridentifikation. Als Muster ist hier die Verteilung der Signalstärken der einzelnen Sensoren aufzufassen. Diese kann man sich in der Auftragung der Amplituden um einen gemeinsamen Mittelpunkt als Stern mit unterschiedlich langen Strahlen vorstellen. In der Regel versucht man, die Dimension des Datensatzes zu reduzieren, indem man anhand der Korrelationen zwischen den Sensoren die Information zusammenzieht. Dies läuft im Wesentlichen auf klassische Verfahren wie Hauptkomponenten- oder Diskriminanzanalyse hinaus, in denen man die Verwandschaft zwischen verschiedenen Profilen anhand von Distanzmaßen, beispielsweise der Mahalanobis-Distanz bemisst. Die Dimensionsreduktion ermöglicht es im Fall, dass zwei Dimensionen ausreichen, verschiedene Muster als Punkte in abgegrenzten Bereichen einer zweidimensionalen Ebene darzustellen.

Ein typischer Vertreter der eNose-Geräte ist die Cyranose $320^{\mathrm{TM}}$ (Smiths Detection Group Ltd, 64 Clarendon Road, Watford, Herts, WD17 1DA, UK). Das Gerät verfügt über 32 Biopolymersensoren und wurde bereits für medizinische Fragestellungen eingesetzt. So konnten Asthmapatienten von gesunden Kontrollprobanden differenziert werden sowie COPD-Patienten von Tumorpatienten
Conclusion: Measuring VOCs with an electronic nose has not yet been standardised and the set-up significantly affects the results. As other researchers use further methods, it is currently not possible to draw generally accepted conclusions. More systematic tests are required to find the most sensitive and reliable but still feasible set-up so that comparability is improved.

[1-3]. Viele methodologische Fragen sind allerdings bislang nicht geklärt, und vor allem gibt es keine einheitliche Methode. Die Luftfeuchte beeinflusst die Sensorsignale massiv, daher scheint auch hier eine Normierung geboten [4].

Ferner ist nicht untersucht, inwieweit die kommerziell erhältlichen eNose-Geräte gleichen Bautyps vergleichbare Ergebnisse liefern, ebenso wenig wie der Einfluss unterschiedlicher Messaufbauten, obgleich diese wesentlich die erhaltenen Daten determinieren. Ziel dieser aus einem Treffen der Arbeitsgruppe deutscher eNose-Anwender entstandenen Arbeit war es, vier verschiedene Cyranose 320-Geräte und drei unterschiedliche Messaufbauten zu vergleichen. Dies soll dem Zweck dienen, die inhärente Komplexität dieser klinisch interessanten Verfahren zu zeigen, die nur auf den ersten Blick einfach erscheinen. Hierdurch soll der Leser auf den gegenwärtigen Stand der Forschung gebracht und darin unterstützt werden, Publikationen zur klinischen Anwendung der eNose mit kritischem Blick zu bewerten.

\section{Methodik \\ $\nabla$}

\section{Probanden}

Wir untersuchten die Atemluft von drei gesunden, nüchternen, nichtrauchenden Probanden (Durchschnittsalter 40 (SD 8) Jahre; 2 männlich, 1 weiblich). Die Probanden boten keinen Hinweis auf ein entzündliches Geschehen zur Zeit der Messung oder eine chronische Atemwegserkrankung.

\section{Elektronische Nase}

Vier Geräte (Hannover, Leipzig, Marburg, München) wurden verwendet und in die drei Aufbauten eingesetzt, die an den Standorten, Leipzig, Marburg und München/Hannover verwendet werden; die Aufbauten wurden zur Durchführung dieses Vergleichs nach Marburg gebracht. In allen Aufbauten wurde die Ausatemluft in Plastikbeuteln aufgefangen (Rossmann ${ }^{\mathrm{TM}}$, Deutschland) und unmittelbar nach Gewinnung über ein einheitlich gewähltes Programm der Cyranose $320^{\mathrm{TM}}$ eingezogen und analysiert (60 s Referenzluft, 60 s Probenluft, 60 s Reinigung). Die Aufzeichnung der Werte erfolgte durch das mitgelieferte Programm PC Nose 6.9.

\section{Design}

- Tab. 1 zeigt ein Schema des Protokolls. Die Probanden 1, 2 und 3 durchliefen die Messaufbauten Leipzig, München/Hannover, Marburg. Alle drei Messaufbauten wurden nach Marburg gebracht und dort aufgebaut. Die eNose-Geräte München, Hannover, Marburg und Leipzig wurden in die Messaufbauten München/Hannover, Marburg und Leipzig eingehängt. Die Temperatur im klimatisierten Messraum betrug konstante $25^{\circ} \mathrm{C}$, die relative Luftfeuchtigkeit lag bei $19 \%$. 
Tab. 1 Darstellung des Versuchsaufbaus. Die Probanden 1, 2 und 3 durchliefen die drei Aufbauten mit den unterschiedlichen Geräten.

\begin{tabular}{|llll|}
\hline Nase & \multicolumn{3}{c}{ Aufbau } \\
\cline { 2 - 3 } & München & Leipzig & Marburg \\
\hline München & $1,2,3$ & $1,2,3$ & $1,2,3$ \\
\hline Hannover & 2,3 & 2 & \\
\hline Leipzig & 2,3 & 2 & \\
\hline Marburg & 2,3 & 2 & \\
\hline
\end{tabular}
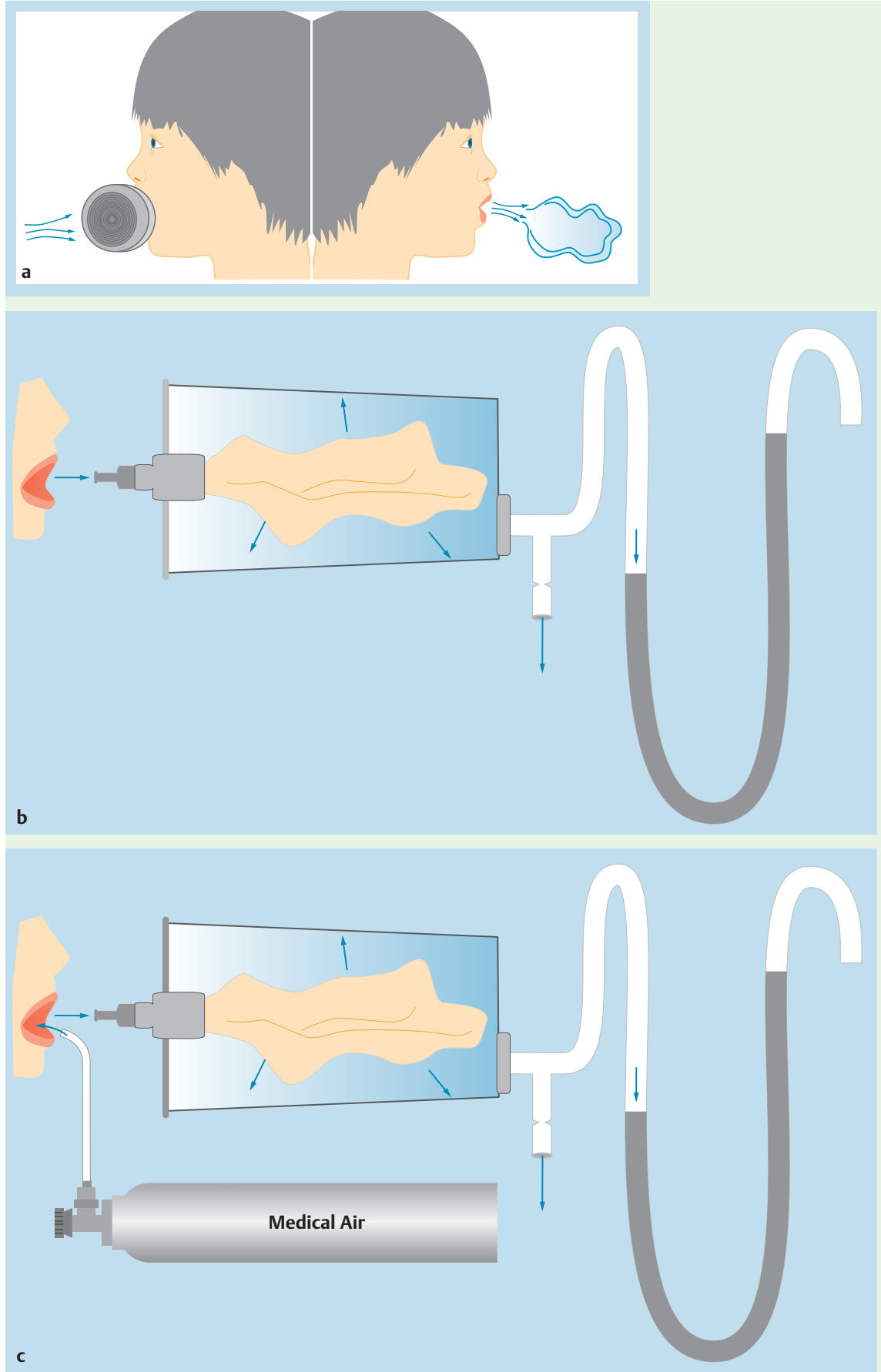

\section{Messaufbauten}

Aufbau Leipzig

Hierbei dient ungefilterte Umgebungsluft als Referenz $(\bullet$ Abb. 1 a). Der Proband atmet einige Male tief über einen kommerziellen Aktivkohlefilter ein. Die sodann in einer tiefen Einund Ausatmung gewonnene Luft wird in einem Sammelbeutel ohne Flusskontrolle gesammelt.

\section{Aufbau München/Hannover}

Als Referenz dient zu 100\% angefeuchtete Umgebungsluft $(\bullet$ Abb. 1b), als Einatemluft normale Raumluft. Die Probanden atmen mit einem konstanten Fluss von $150 \mathrm{ml} / \mathrm{s}$ über $10 \mathrm{~s}$ in den Sammelbeutel in einem bag-in-box-System aus. Der in Hannover verwendete Aufbau ist identisch mit dem aus München, daher wurde zur Prüfung nur die Apparatur München eingesetzt.

Abb. 1 a Beim Aufbau Leipzig wird Umgebungsluft durch einen Aktivkohlefilter eingeatmet und anschließend in einen Plastikbeutel ausgeatmet, um dann direkt mit der Cyranose $320^{\mathrm{TM}}$ gemessen zu werden. Die Referenzluft ist ungefilterte Umgebungsluft.

b Beim Aufbau München/Hannover wird ein Sammelbeutel (der gleiche wie beim Aufbau Leipzig) in einem bag-in-box-System zum Sammeln des Expirats verwendet. Ein Wassermanometer mit Markierungen dient dem Probanden zur Sicherstellung eines konstanten exspiratorischen Flusses von $150 \mathrm{ml} / \mathrm{s}$. Die Referenzluft ist zu $100 \%$ befeuchtete ungefilterte Umgebungsluft.

c Beim Aufbau Marburg wird der Aufbau München/ Hannover mit konstantem exspiratorischem Fluss, dem Sammelsystem, bestehend aus Plastikbeutel und Box, derart ergänzt, dass standardisierte medizinische Luft eingeatmet sowie zu $100 \%$ befeuchtete medizinische Luft als Referenz verwendet wird. 
Tab. 2 Berechnete Varianzen der ersten Hauptkomponente der verschiedenen Aufbauten mit Standardabweichung.

\begin{tabular}{|llll|}
\hline 1. Hauptkomponente & \multicolumn{3}{c|}{ Aufbau } \\
\cline { 2 - 4 } & München & Leipzig & Marburg \\
\hline Varianz & 0,0000385 & 0,0030636 & 0,0000257 \\
\hline Standardabweichung & 0,0062070 & 0,0553500 & 0,0050650 \\
\hline
\end{tabular}

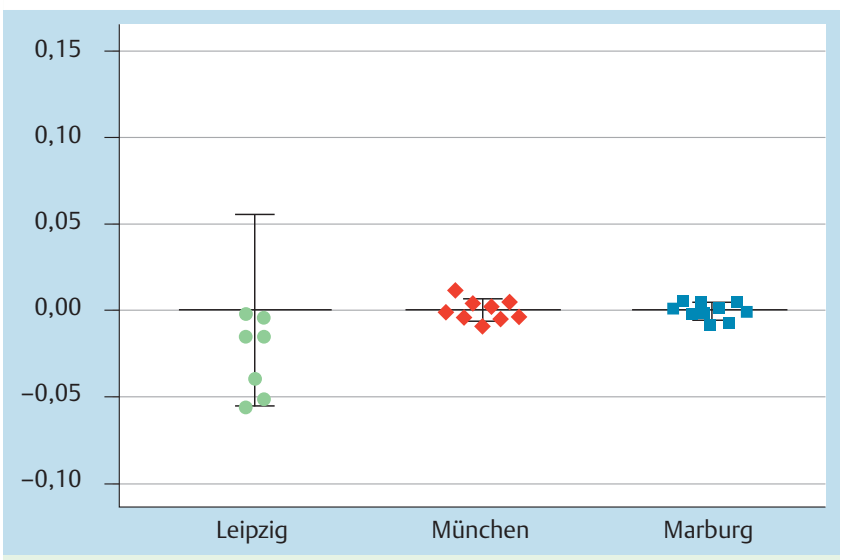

Abb. 2 Grafische Darstellung der Varianzen der 1. Hauptkomponente (maximale Komponente der Gesamtvarianz) der Aufbauten Leipzig, München/Hannover und Marburg.

\section{Aufbau Marburg}

Dieser ( $\bullet$ Abb.1 c) entspricht dem Aufbau München/Hannover mit dem Unterschied, dass keine Umgebungsluft verwendet wird. Es wird medizinische Luft (Aer medicinale, Linde, Deutschland) über ein Tauchdemandventil eingeatmet; diese Luft dient (100\% angefeuchtet) auch als Referenzluft.

\section{Datenanalyse}

Die Auswertung erfolgte mittels der Widerstandsänderungen der 32 Resistoren, entweder anhand der vom Gerät ausgegebenen Werte (Marburg, München/Hannover) oder anhand der Echtzeitmesswerte durch ein separates Programm (Leipzig). Alle Daten konnten in der Hauptkomponentenanalyse sehr gut durch die drei größten Komponenten beschrieben werden, die zusammen 99\% der Varianz abdeckten. Zum Vergleich der eNose-Geräte wurden alle drei Probanden mit allen vier Geräten am Aufbau München/Hannover gemessen. Zum Vergleich der Aufbauten wurden alle drei Probanden mit der eNose München am Aufbau München/Hannover gemessen. Zur statistischen Trennung der verschiedenen Aufbauten diente eine lineare Diskriminanzanalyse (LDA) der Hauptkomponenten. Dies ergab den Prozentsatz der korrekt dem jeweiligen Messaufbau zugeordneten Probanden. Mittels reduzierter Trainingssets und anschließender Zuordnung über Validierungssets wurde das Ergebnis bewertet (Kreuzvalidierung). Als Distanzmaß diente die Mahalanobis-Distanz der Versuchsaufbauten zueinander, die bedingt durch wenige Patienten mithilfe einer MCD-Näherung (Minimum Covariance Determinant) berechnet wurde. SD steht für die Standardabweichung.
Tab. 3 Varianzen mit Standardabweichung der ersten Hauptkomponente aller Geräte und aller Aufbauten.

\begin{tabular}{|lll} 
1. Hauptkomponente & Nase & Aufbau \\
Varianz & 0,00042 & 0,00895 \\
\hline Standardabweichung & 0,02054 & 0,09459 \\
\hline
\end{tabular}

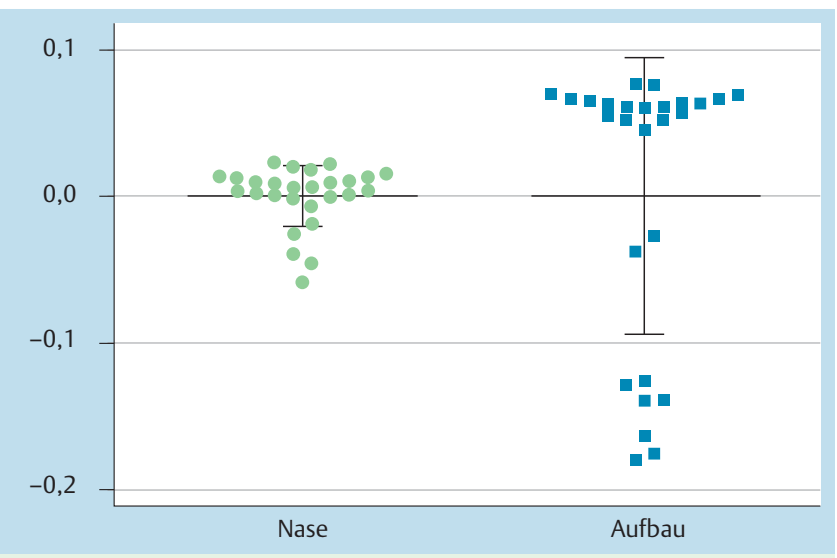

Abb.3 Vergleich der Varianzen der vier eNose-Geräte und der Aufbauten untereinander.

\section{Ergebnisse}

Beim Vergleich der Aufbauten ergaben sich deutlich verschiedene Gesamtvarianzen der Messwerte. Der Aufbau Leipzig zeigte eine Varianz von 0,003 (SD 0,055), der Aufbau München/Hannover von 0,000039 (SD 0,006) und der Aufbau Marburg von $0,000026(S D 0,005)(\bullet$ Tab. 2, $\bullet$ Abb. 2). Der Vergleich der Aufbauten ergab eine Varianz von 0,009 (SD 0,096). Verglich man die baugleichen Geräte am Aufbau München/Hannover, zeigte sich eine Varianz von 0,0004 (SD 0,0205) ( $\bullet$ Tab. 3, ๑ Abb. 3)

Die Hauptkomponentenanalyse erwies eine gute statistische Trennbarkeit der Messaufbauten mit versus ohne Befeuchtung der Referenzluft im 3D-Plot ( $\bullet$ Abb.4) Die Diskriminanzanalyse ergab bei der Trennung des Aufbaus Leipzig gegen München/ Hannover eine Genauigkeit von 90,1\%, Leipzig gegen Marburg 89,5\% und Marburg gegen München/Hannover 72,8\% ( $\bullet$ Tab. 4). Die Mahalanobis-Distanz zwischen den Aufbauten Leipzig und München/Hannover betrug 18,5 (MCD-Wert), zwischen Leipzig und Marburg 18,6 (MCD-Wert) und zwischen München/Hannover und Marburg 1,8 (MCD-Wert) ( $\bullet$ Tab.4).

\section{Diskussion \\ $\nabla$}

Beim Vergleich von drei Messaufbauten zur Exhalatanalyse mittels elektronischer Nase zeigte sich, dass diese einen deutlichen Einfluss auf die Ergebnisse der Messung von gesunden Probanden hatten. Vor allem resultierten sie in unterschiedlichen Varianzen der Messungen. Hierbei ergab der Versuchsaufbau Marburg die geringste Varianz, der Versuchsaufbau Leipzig die höchste.

In der Zusammenschau waren die Muster der Sensorsignale verschieden. So konnten die Messaufbauten im 3D-Plot sicher getrennt werden. Die Mahalanobis-Distanzen, die ein Maß des sta- 


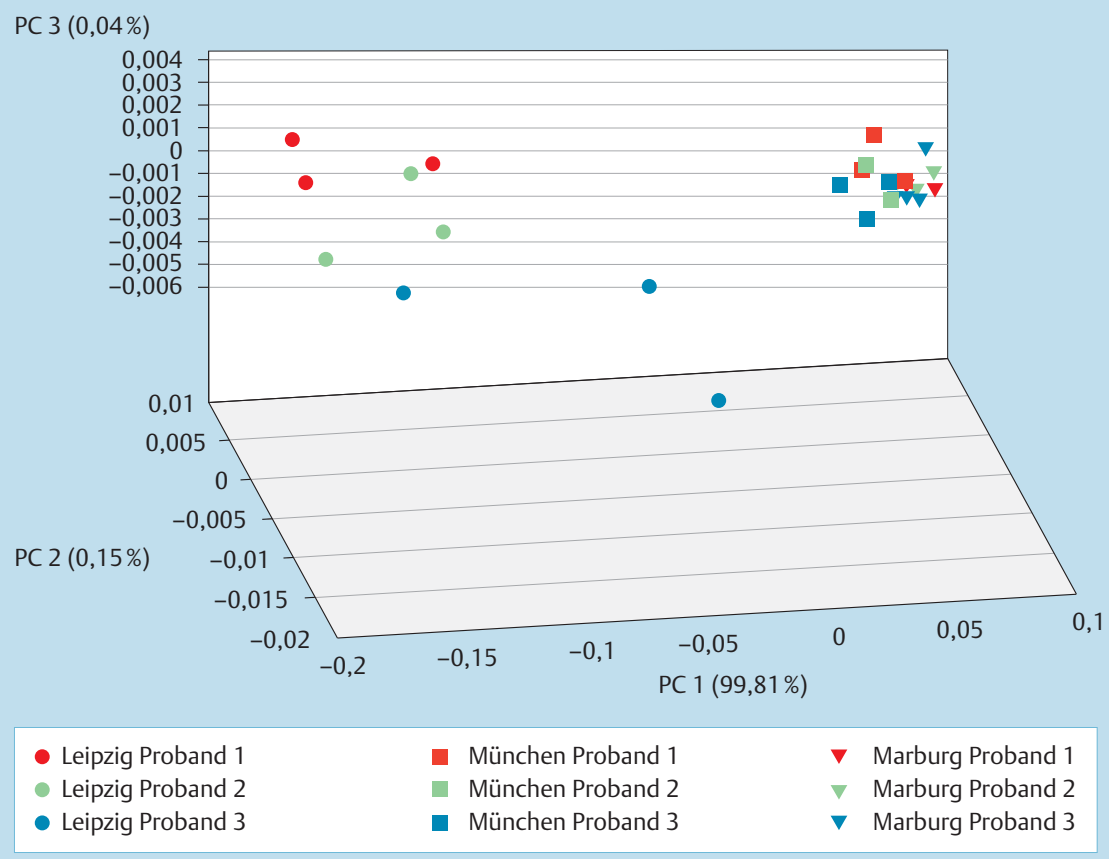

Abb. 4 3D-Plot der ersten drei Hauptkomponenten der Messungen mit den unterschiedlichen Aufbauten.
Tab.4 Mahalanobis-Distanzen (und Validierungswert) zwischen den unterschiedlichen Aufbauten.

\begin{tabular}{|lll} 
& München & Marburg \\
\hline Leipzig & $18,532(90,12 \%)$ & $18,646(89,51 \%)$ \\
\hline München & & $1,820(72,84 \%)$ \\
\hline
\end{tabular}

tistischen Abstands darstellen, trennten vor allem den Aufbau Leipzig von denjenigen aus Marburg und München/Hannover. Die Exhalatmuster sind demnach stark abhängig von der Art des Messaufbaus. Hierin äußern sich vermutlich zum Teil Unterschiede des Sammelsystems und der Flusskontrolle, vor allem jedoch solche der unterschiedlichen Behandlung der Einatemluft und Referenzluft. Es wurde bereits gezeigt, dass die exspiratorischen Widerstände bzw. Flussraten die VOC-Profile beeinflussen können [1]. Zur Varianzreduktion empfiehlt es sich daher, einen konstanten exspiratorischen Fluss vorzugeben.

Allerdings zeigten auch baugleiche Geräte im gleichen Messaufbau geringfügige Unterschiede; dies ist durch Unterschiede der Sensoren in der Fertigung oder Alterungsprozesse erklärbar. Um dies zu prüfen, müssten u.a. bei konstanten Bedingungen standardisierte Gasgemische untersucht werden. Ferner sind innerhalb des Zeitrahmens der Untersuchung ebenfalls Veränderungen der VOC-Zusammensetzung aufgrund individueller Variation oder zirkadianer Rhythmik denkbar.

Gemäß den bislang publizierten Daten zur Cyranose 320 ${ }^{\mathrm{TM}}$ können Patienten mit COPD gut von Patienten mit Asthma sowie Asthmatiker von Gesunden statistisch unterschieden werden $[1,3]$. Der Messaufbau, der für diese Versuche verwendet wurde, unterschied sich in einigen Punkten von den hier getesteten Aufbauten. Vor allem fanden ein inspiratorischer Aktivkohlefilter sowie eine Feuchtereduktion des Exhalats über Silikagel Anwendung. Die von uns in Vorversuchen durchgeführten umfänglichen Analysen erwiesen es als unmöglich, die Feuchtigkeit komplett oder auch nur reproduzierbar aus dem Exhalat zu eliminieren. Ferner zeigte sich, dass alle Sensoren der Cyranose $320^{\mathrm{TM}}$ stark auf Luftfeuchte ansprechen, jedoch in unterschiedlichem
Maße und mit unterschiedlichen Kennlinien. Aufgrund dieser Tatsache wurde - entgegen der Feuchtigkeitsreduktionsmethode von Fens et al. und Dragonieri et al. [2,3] - in den Messaufbauten Marburg und München/Hannover die Referenzluft auf 100\% befeuchtet. Dies diente dazu, ihre Feuchte derjenigen der Atemluft anzupassen, die feuchtigkeitsbedingten Widerstandsänderungen gleich zu halten und die auf VOCs zurückgehende Widerstandsänderung unverfälscht zu erfassen. Ferner wurde im Messaufbau Marburg medizinische Luft zur Einatmung verwandt, um den Einfluss der in der Umgebungsluft vorhandenen VOCs zu reduzieren; diese äußerte sich in einer verringerten Varianz.

Als Nachteil der Aufbauten Marburg und München/Hannover erschien der komplexere Aufbau, der einen schnellen Transport des Systems zum Patienten limitiert und die Kosten und den Wartungsaufwand des Systems erhöht. Der Aufbau Leipzig ist vergleichsweise einfach und gewährleistet einen schnellen Transport des Systems zum Patienten. Als Referenz wurde Umgebungsluft verwendet, die weder feuchtigkeitsreduziert noch mit Feuchtigkeit angereichert war. Die Ausatmung erfolgte ohne Flusskontrolle. Zur Reduktion von VOCs der Umgebungsluft erfolgte die Einatmung über einen Aktivkohlefilter. Die Varianz, aber auch die Signalstärke der so erhobenen Daten war deutlich höher als in den Messaufbauten mit künstlich angehobener Feuchtigkeit in der Referenzluft. Inwieweit das die Diskriminierungsfähigkeit günstig beeinflussen kann, müssen weitere Untersuchungen auch an kranken gemessenen Personen zeigen.

Inwieweit die Unterschiede der Messaufbauten die Fähigkeit zur statistischen Erkennung von Patientengruppen oder induzierten Änderungen beeinflusst, können nur weiterführende Experimente zeigen. Aus den Ergebnissen lässt sich jedoch schlussfolgern, dass unterschiedliche Messaufbauten die Vergleichbarkeit der Daten in hohem Maße beeinträchtigen. Hier erscheint vor allem die unterschiedliche Behandlung der Einatemluft und der Referenzluft bedeutsam. Es ist ungeklärt, welche der Methoden den Vorzug verdient. Dies gilt umso mehr, als unterschiedliche Messverfahren auch unterschiedliche Sensitivitäten und daher unterschiedliche Störeinflüsse involvieren. Die Cyranose 320 kann als eher wenig empfindliches Gerät gelten, das nur im 
ppm-Bereich hinreichend sensitiv ist. Die systematische Untersuchung dieser Fragen sollte der Erstellung nationaler oder besser internationaler Empfehlungen vorangehen, um unproduktive Entwicklungen zu vermeiden.

\section{Interessenkonflikt}

$\nabla$

Die Autoren geben an, dass kein Interessenkonflikt besteht.

\section{Literatur}

1 Dragonieri S, Schot R, Mertens BJ et al. An electronic nose in the discrimination of patients with asthma and controls. J Allergy Clin Immunol 2007; 120: 856

2 Dragonieri S, Annema JT, Schot $R$ et al. An electronic nose in the discrimination of patients with non-small cell lung cancer and COPD. Lung Cancer 2009; 64: 166

3 Fens N, Zwinderman AH, van der Schee MP et al. Exhaled breath profiling enables discrimination of chronic obstructive pulmonary disease and asthma. Am J Respir Crit Care Med 2009; 180: 1076

4 Dressel H, Hofbauer J, Schierl R et al. Measurement of exhaled breath samples using an electronic nose: methodological issues. ERJ 2009; Poster No 3951 